\title{
Thematic and Episodic Framing of Depression: How Chinese and American Newspapers Framed a Major Public Health Threat
}

\author{
By Yuan Zhang* \\ Yan $\mathrm{Jin}^{\dagger}$
}

\begin{abstract}
Given the health threat of depression in China and the US, as well as the importance of media framing for public understanding of the issue, this paper comparatively analyzed how Chinese and American newspapers thematically and episodically framed depression from 2000 to 2012. Grounded in the theoretical framework of frame building, we also attempted to shed light on how cultural values and organizational constraints influenced the ways in which media framed depression. Results showed that Chinese newspapers framed depression more thematically than American newspapers. American newspapers of national influence used more thematic framing than newspapers of regional/local influence. Despite an overall tendency of thematic framing, Chinese newspaper framing of depression became increasingly episodic over time. Implications for framing research and mental health communication are discussed.
\end{abstract}

Keywords: Chinese and American newspapers, comparative content analysis, depression, news framing

\section{Introduction}

Depression is one of the most commonly diagnosed mental illnesses worldwide; by 2020, it will become the second leading cause of chronic disability, trailing only ischemic heart disease (Johansson, Bengs, Danielsson, Lehi, \& Hammarstrom, 2009; Metzl \& Angel, 2004). In the US, major depression is the leading cause of disability for Americans between the ages of 15 and 44 (Stats and Facts about Depression in America, 2015). About 6.7\% of American adults suffer from it each year and $3.3 \%$ of 13 to 18 year olds have experienced a seriously debilitating depressive disorder (Depression, 2014). Only about half of the sufferers have received medical treatment or service (Wang et al., 2005).

In China, unipolar major depression affects approximately $9 \%$ of the adult population and 15-30\% of Chinese adolescents (Ryder, Sun, Zhu, Yao, \& Chentsova-Dutton, 2012). By 2020, various forms of mental disorders including depression will constitute the largest burden on public health in China (He \& Wang, 2012). In recent years, the number of people who suffer from depression has been steadily increasing (Zhao, 2009). Additionally, more than 200 thousands depression sufferers commit suicide each year (CCTV-China Central Television, 2015). Perhaps the most alarming statistic to date comes from a recent China Central Television report estimating a total of 90 million sufferers at the end of 2015 (CCTV-China Central Television, 2015). However, depression only started

\footnotetext{
* Assistant Professor, Westfield State University, USA.

${ }^{\dagger}$ Associate Professor, University of Georgia, USA.
} 
to be recognized and diagnosed in Chinese hospitals in recent decades (Mao, Tang, \& Cai, 2003). As late as 1996, 95\% of the sufferers did not receive any diagnosis or treatment (Murray \& Lopez, 1996). Longitudinal data show that a lack of knowledge among sufferers, caregivers, and even medical professionals led to the alarmingly low diagnosis rate of $10 \%$ and treatment rate of $1 \%$ (Tan, 2004). To this day, significant cultural (Ryder et al., 2012) and historical (Kleinman, 1986, 1995) barriers remain for the recognition, prevention and treatment of depression within the Chinese population.

The news media can not only bring depression into public discourse, but also define and frame the issue in ways that may help alleviate stigmas, increase public understanding and enhance mental health literacy. In particular, framing issues thematically (i.e., highlighting systemic and societal aspects of issues) or episodically (i.e., emphasizing isolated and personal aspects of issues) can have direct effects on public attitudes and perceptions (Gross, 2008; Iyengar, 1991). Prior research suggests that news media operating in different cultures and media systems may have different framing tendencies (Hook \& Pu, 2006). Western news media, for instance, have been found to frequently use episodic framing to cover social problems (Kim, Carvalho, \& Davis, 2010). By focusing on isolated events and personal stories, episodic framing can lead the public toward individualized causes and solutions while overlooking societal dimensions and policy-based remedies in addressing social problems (Price, Tewksbury, \& Powers, 1997; Shah, Kwak, Schmierbach, \& Zubric, 2004). In the case of mental health issues, individualized responsibility attribution can also reinforce existing negative public perceptions and stigmatization (Schnittker, 2008; Walker \& Read, 2002). As such, news media's thematic and episodic framing of mental health issues including depression can have important implications for public knowledge, perception, responsibility attribution and public opinion support for various solution alternatives of the issues on hand.

The ways in which news media frame issues may be a function of various macro- and micro-level frame building factors, in particular cultural values and organizational constraints (Kim et al., 2010; Scheufele, 1999). Prior research examining media coverage of mental health issues has dealt extensively with its stigmatizing representation and effects (for a review, see Stout, Villegas, \& Jennings, 2004). Few have carried out a systematic and comparative investigation of how news media in different cultures and media systems frame mental health issues in thematic and episodic approaches. American and Chinese news media operate within drastically different media systems and two distinct cultures that come from the opposite ends of the individualism-collectivism cultural spectrum. They provide an ideal case for exploring the potential impact of cultural and organizational factors on the frame building of depression-related communication. This study therefore compares depression coverage in Chinese and American print news media by content-analyzing how newspapers in the two countries framed depression thematically and episodically in the most recent past decade. 


\section{Literature Review}

\section{News Framing and its Public Opinion Implications}

When covering complex issues, journalists tend to rely on news frames to help present large amounts of information in easy-to-understand interpretative packages (Kim \& Willis, 2007). In doing so, they inevitably highlight certain aspects of an issue and make them more salient than others (Entman, 2004). A frequently seen news framing tendency involves thematic vs. episodic approaches (Gross, 2008).

Thematic framing refers to the portrayal and presentation of issues through information about their systemic causes, trends and consequences (Iyengar, 1991). In covering public health problems, thematic framing often includes expert analyses of contextual conditions, systematic evidence of widespread outcomes, statistical trends of morbidity and mortality, reports on research in disease prevention, intervention and treatment, discussion of risk factors and their social indicators, and presentation of community-level and policy-based remedies (Kim \& Willis, 2007; Lawrence, 2004). Research has shown that thematic framing can influence people's thoughts and feelings about social problems (Iyengar, 1991; Price et al., 1997; Shah et al., 2004). Health advocates believe that by providing adequate background information, contextual conditions and widespread consequences, thematic framing has the potential to improve public understanding of systemic (vs. isolated) risk factors, encourage public support for collective (vs. individual-level) solutions, as well as alleviate stigmas that are usually caused by and associated with blaming and demonizing individual sufferers.

However, existing research suggests that episodic framing may be more prevalent in news coverage of various social issues including foreign relations, poverty and abuse (Hook \& Pu, 2006; Kim et al., 2010; Kunkel, Smith, Suding, \& Biley, 2006; Mastin, Choi, Barboza, \& Post, 2007). Episodic framing refers to the portrayal and presentation of issues through either a specific event that serves as a representative anecdote of the broader issue or the life story of a person who could put a face on the issue (Iyengar, 1991). While episodic framing may achieve some exemplification effects in reaching news audiences (Zillmann, 2002), it may also have unintended consequences that potentially hinder issue understanding and responsibility attributions (Major, 2009). In particular, an overemphasis on anecdotal events and personal stories may condition the public to "describe chronic problems such as poverty and crime not in terms of deep-seated social or economic conditions, but as mere idiosyncratic outcomes," causing news audiences to hold individuals responsible for causing and fixing social problems (Iyengar, 1991, p. 136). Putting individuals at the center of discourse and focusing on deficiencies in and modifications of individual-level risk behaviors and lifestyles that are dissociated from larger social context, episodic framing can divert public attention away from systemic flaws in political, social, and economic environments, and downplay the importance of society-level remedies such as policy changes, regulatory efforts, and correction of social inequalities and injustices (Kim et al., 2010). 
In short, the ways in which the media frame issues thematically and/or episodically can have a significant impact on how the general public understands the issue and anticipates solutions. Before analyzing how news media in different cultures and media systems frame depression, it is necessary to examine some key factors that potentially influence news framing.

\section{Cultural and Organizational Influences on News Framing}

Scholars have attempted to understand why the media frame issues in certain ways (Scheufele, 1999). Several frame-building factors have been identified, among which social norms/cultural values and organizational pressures/constraints may be particularly useful in understanding why news media from different cultures and/or media systems frame issues in different ways.

Cultural values influence the way people communicate (Triandis, 1995), which in turn may affect how the media frame issues. Collectivistic cultures embrace "contradictions among objects in a yin-yang field of constant change," thus communication tends to focus less on specifics and more on contexts (Gries \& Peng, 2002, p. 175). As a result, thematic news framing in which an issue is placed in a larger social context may be preferred (Hook \& Pu, 2006). In contrast, Western cultural reasoning is driven by formal logic, and communication in individualistic societies tends to focus on specific objects and categories (Gries \& Peng, 2002). Consequently, Western media may prefer episodic framing in which issues are presented as specific events or individual cases (Kim et al., 2010).

Organizational characteristics, such as media political and/or business orientations, and target audience (e.g., a national vs. regional audience), may also influence issue framing (Dorfman, Woodruff, Chavez, \& Wallack, 1997; Gans, 1979; Shoemaker \& Reese, 1996). For instance, journalists racing against deadlines in commercial media may prefer episodic framing which requires less time investment in background research and data collection than thematic framing (Iyengar, 1991). Moreover, episodic framing in forms of event coverage and narrative storytelling not only simplifies the storyline, but also attaches a human face to the issue. By personifying and exemplifying issue significance, episodic framing helps to attract news audience and serves the commercial interests of privately owned media organizations (Kim, Carvalho, Davis, \& Mullins, 2011; Zillmann, 2002).

\section{Thematic and Episodic Framing of Health Issues}

As some of the most pressing social problems in modern societies, public health issues such as obesity and mental health have received much media attention (Conrad, 2001; Kim \& Willis, 2007; Stout et al., 2004). While existing research has yet to examine media framing of depression in different cultures and the influence of key frame building factors, scholars have indeed looked into thematic and episodic framing of various other public health issues, especially in the context of US news media (both newspapers and television). Focusing on the television medium, Dorfman et al. (1997) examined how local 
TV news in California framed the issue of youth violence, and found that thematic framing with references to broader social factors was used less frequently than episodic framing. Similarly, a qualitative analysis of $28 \mathrm{TV}$ news stories about postpartum disorders aired from 2000 to 2007 revealed that TV news was engaged in a process of individualizing and decontextualizing a social problem through the use of journalistic routines such as telling personalized anecdotal stories (Dubriwny, 2010). On the other hand, breast cancer coverage aired in the three major broadcast TV networks from 1974 to 2003 displayed an increasing pattern of thematic framing over time (Cho, 2006). In terms of newspaper framing, an analysis of avian flu coverage in four major US newspapers revealed that the coverage was dominated by episodic framing (Dudo, Dahlstrom, \& Brossard, 2007).

Research investigating how Chinese news media thematically and episodically frame health problems is relatively scarce. However, the limited amount of existing research indicates that Chinese health news framing may be more thematic than episodic. When covering the 2003 SARS outbreak, People's Daily, China's largest daily newspaper, emphasized "the social dimensions of the disease" (Wang, 2007, p. 29). Meanwhile, HIV/AIDS coverage in the same newspaper from 1986 to 2002 ignored individual cases of sufferers, "making them impalpable in the news discourse" (Dong, Chang, \& Chen, 2008, p. 371). Similar conclusions may be drawn from a limited amount of research comparing health coverage in China and the US. When covering SARS, the Associated Press (AP) used more human interest frames to tell personal stories than China's Xinhua News Agency (Beaudoin, 2007). Similarly, AP featured more personal story frames than Xinhua did when covering breast cancer in China (Liu, Ley, \& Brewer, 2011). Taken together, these studies suggest that episodic framing as a journalistic storytelling device routinely used in Western news media may be less prevalent in Chinese media.

The preceding brief review revealed several areas that need more research attention. First, there is a clear lack of research on media framing of depression, despite the fact that it is rapidly becoming one of the most severe public health threats worldwide as well as in China and the US. Existing research in mental health coverage mostly focused on how the media present a stigmatizing portrayal of mental disorder sufferers as violent, dangerous and unpredictable (Stout et al., 2004). However, little research has examined how the media frame the issue of depression thematically and episodically. Given the potential public opinion and health policy implications of thematic and episodic news framing, this study offers a comparative content analysis focusing on how Chinese and US newspapers framed this critical public health issue in the most recent past decade.

Second, while framing research has extensively examined what frames the media tend to use and what effects they have on audience responses, the question of frame building, or what factors influence news framing, remains underinvestigated (Kim et al., 2010). This study explores how two key macro-level frame building factors, cultural values and organizational constraints, potentially influence news framing of depression. Chinese and US news media, coming from 
the opposite ends of the individualism-collectivism cultural spectrum and operating within drastically different media systems, provide a meaningful empirical case for addressing this theoretical question in news framing.

\section{Hypotheses and Research Questions}

As reviewed above, the cross-cultural theory of individualism-collectivism and the media frame building theory indicate that cultural values may be an important frame building factor shaping the thematic and episodic framing of news coverage in different cultures. Empirical evidence also suggests that US news media may be more likely to frame issues episodically whereas Chinese news media may lean toward thematic framing. Therefore, comparatively speaking:

H1: Chinese newspapers will frame depression more thematically than US newspapers.

Media frame building theory also suggests the influence of organizational characteristics on news framing. Due to a lack of empirical research examining the impact of this frame building factor on depression framing in national and local newspapers, this study queries:

RQ1a: Were there any differences between Chinese national and local newspapers in the use of thematic and episodic framing when covering depression?

RQ1b: Were there any differences between US national and local newspapers in the use of thematic and episodic framing when covering depression?

Additionally, we attempted to detect longitudinal changes in news framing of depression:

RQ2a: How did US newspaper framing of depression change from 2000 to 2012 ?

RQ2b: How did Chinese newspaper framing of depression change from 2000 to 2012 ?

\section{Method}

\section{Sample}

This study used newspapers as the sampling universe, as they continue to be some of the main venues for disseminating health information and setting media agenda in both China and the US (Luther \& Zhou, 2005; Peng \& Tang, 2010). To obtain the Chinese data, the China Core Newspaper Full-text Database was used. This database archives, on a daily basis, full-text news items from 1,000 newspapers published in mainland China since 2000. Using the keywords of 抑有阝 
(depressed) and 抑有症 (depression) ${ }^{1}$, the most commonly used terms in both medical and non-medical Chinese literature on depression (Guo \& Guo, 2005; Tian \& Chen, 2014), we retrieved 1,507 news items published from 2000 to $2012^{2}$ in a wide variety of newspapers, including national and local newspapers, statecontrolled Party newspapers and market-oriented City newspapers, and general and special interest newspapers. Using a filtering procedure (for details of the procedure, see description of sampling for the US data), we removed 431 redundant and/or irrelevant items. Systematic sampling by drawing every second item from the remaining 1,036 items resulted in a sample size of 538. During pretesting, nine additional items that fell into any one of the filtering categories were further removed, bringing the sample size down to 527. Among them, 404 were from national newspapers (e.g., People's Daily) and 123 from local newspapers (e.g., Beijing Daily). To achieve comparable sample sizes for the national and local newspaper data, systematic sampling was conducted by drawing every fourth item from the 404 national newspaper articles. The final Chinese sample consisted of 224 news items, including 101 from national newspapers and 123 from local newspapers.

To obtain the US data, two newspapers with national influence, readership and distribution mechanisms were selected: New York Times and USA Today. To select newspapers with local influence, readership and distribution, the Census Bureau's classification of US regions was used: the Northeast, the South, the Midwest, and the West (Census Regions and Divisions of the United States, 2012). From each region, one newspaper was selected based on its circulation size, local presence (measured by number of local offices and staffers), and availability in the LexisNexis database: Philadelphia Inquirer (Northeast), Houston Chronicle (South), Star Tribune (Midwest), and Denver Post (West). In order to match the sampling timeframe of the Chinese data, the US data sampling timeframe was also set for 2000-2012.

Searches in the LexisNexis database were conducted using the keywords of "depression," "depressed, or "bipolar disorders" appearing in news headlines or index terms. After sorting through the full text of each news item, those that fell into the following categories were excluded: 1) items unrelated (e.g., stories about the Great Depression or tropical depression) or marginally related to depression (e.g., stories that mentioned depression in passing but did not treat the topic in a substantive way); 2) duplicate items (e.g., the same story published in a different edition of the same newspaper); 3) web items which were mere rewrites of their print versions; 4) stories carried verbatim from wire services and/or other media organizations/outlets; and 5) non-news items (e.g., letters to the editor, wedding/engagement announcements, obituaries, weather, sports, traffic, calendar, etc.). Systematic sampling of the remaining items resulted in a final sample of 228 newspaper articles for the US data.

\footnotetext{
${ }^{1}$ The keyword of 躁郁症 (bipolar disorders) was initially used in the search. However none of the yielded items included this term. We then removed it from the list of search terms.

2 The year 2000-2012 was chosen for the Chinese sample since considerable amount of depression coverage only started to emerge around early 2000 . The same sampling time frame was used for the American data to ensure comparability.
} 


\section{Measures and Reliability}

Measurement of news framing was developed based on: 1) a review of theoretical and empirical work and adaptation of existing measures (e.g., Iyengar, 1991); and 2) an inductive examination of the depression coverage in the sample. Thematic framing refers to framing that places an issue in a larger and more abstract social context. The primary focus is on providing contextual and bigpicture information. This framing approach may use historical data, statistics, and expert interviews. The overall appeal is on providing objective information. The communication purpose is to encourage audience cognitive information processing. Episodic framing involves storytelling in which an issue is presented in a specific event or case. The primary focus is on telling personal stories, describing anecdotal events, and/or presenting individual cases. This framing approach may use typical storytelling techniques such as characters, plots, and the development and resolution of conflicts. The overall appeal is on human interest. The communication purpose is to generate audience affective responses.

News framing was measured on a 1-to-5 ordinal scale (1=episodic framing only, $2=a$ combination of episodic and thematic with an emphasis on episodic framing, $3=$ =qual emphasis on episodic and thematic framing, $4=a$ combination of episodic and thematic with an emphasis on thematic framing, 5=thematic framing only). The relative weight of episodic vs. thematic framing, when both were identified in a news item, was determined based on the length (space/time) of the use of each framing as well as the gestalt (overall) impression that the news item left on the reader/viewer. For the US data, a pretest in which two coders both coded a randomly selected $10 \%$ of the sample yielded a Cohen's kappa of 0.793 $(p<0.001)$. For the Chinese data, a pretest in which two coders both coded a randomly selected $10 \%$ of the sample yielded a Cohen's kappa of 0.848 $(p<0.001)$. After establishing beyond-chance inter-coder reliability, coders independently coded the data by examining the full texts of all news articles.

\section{Results}

Table 1 provides the mean framing scores of Chinese and US newspapers' depression coverage. Between-culture comparison using the independent-samples t-test procedure showed that Chinese newspaper framing was more thematic $(M=4.29, S D=1.05)$ than US newspaper framing $(M=3.89, S D=1.5), t(450)=3.312$, $p<0.01$, supporting H1.

RQ1a and RQ1b explore differences in the extent of episodic and thematic framing between national and local newspapers in China and the US. No significant difference was found in the Chinese sample. On the other hand, US national newspapers $(M=4.41, S D=1.08)$ put more emphases on thematic framing than local newspapers $(M=3.65, S D=1.59)$. An independent-samples t-test showed that this difference was statistically significant, $t(226)=4.192, p<0.001$ (Table 1).

RQ2a and RQ2b examine changes over time in Chinese and US newspaper framing of depression. A nonparametric correlation analysis looking into the relationship between year of publication and news framing showed that from 
2000 to 2012, Chinese newspaper framing of depression has become less thematic and more episodic ( $r h o=-0.238, p<0.001$ ). US newspaper framing of depression did not show any discernible change over time.

Table 1. Depression Framing by Chinese and US Newspapers, 2000-2012

\begin{tabular}{|l|c|c|c|}
\hline \multicolumn{4}{|l|}{ Newspaper Reach } \\
\hline Country & National Papers & Local Papers & Overall \\
\hline China & 4.21 & 4.20 & 4.29 \\
\hline United States & 4.41 & 3.65 & 3.89 \\
\hline
\end{tabular}

Note: Entries are average framing scores based on a scale of 1-5. 1=episodic framing only, 2=a combination of episodic and thematic with an emphasis on episodic framing, $3=$ equal emphasis on episodic and thematic framing, $4=a$ combination of episodic and thematic with an emphasis on thematic framing, $5=$ thematic framing only.

\section{Discussion}

Depression is rapidly becoming one of the most severe public health threats worldwide, especially in China and the US. This study comparatively analyzed how Chinese and US newspaper coverage framed depression from 2000 to 2012. Results suggest that diverging cultural values (e.g., collectivist vs. individualistic values) and varying organizational characteristics (e.g., nationally vs. regionally oriented newspapers) may have influenced the different ways in which newspapers framed depression thematically and episodically.

\section{More Thematic Framing in Chinese than in American Newspapers}

As predicted, Chinese newspaper coverage of depression was more thematic than US newspapers. Compared to Western news media that routinely use episodic framing to tell personal stories, Chinese newspapers focused less on attaching a human face to a larger issue. Rather, they frequently emphasized broad themes and big-picture scenarios such as cultural taboos in discussing depression in the larger society, stigmas existing in the social environment, or the lack of mental healthcare in the public health system. Typical news articles covered general facts and statistics to a greater extent than specific stories from individual sufferers. When personal stories did get media attention, they were more likely to be covered as unnamed patient cases focusing on medical diagnostic and treatment information rather than individualized stories with characters, storylines, and affective appeals.

These findings provide empirical support for the media frame building thesis, suggesting that key macro-level factors such as cultural values and organizational constraints may be at play in the frame building process of depression coverage in China and the US. For example, Chinese journalists are likely influenced by the collectivistic communication style of emphasizing contexts over particulars. Collectivistic cultures also tend to value the collective over the individual, viewing the latter as part of a larger social group and emphasizing group well-being over individual needs and interests (Triandis, 1995). In terms of organizational 
influences, the political agenda of the Party-state to highlight positive aspects of an issue and downplay negative personal impact may compel media organizations to adopt the practice of "the invisible victim" (i.e., absence of personal stories) in health news coverage (Dong et al., 2008). As a result, depression coverage seemed to have repeated the pattern of less episodic and more thematic framing found in the coverage of a host of other public health issues identified in existing research.

Conversely, American news media have long been considered as leaning toward episodic framing, arguably due to the deep-seated individualistic cultural propensity to tell personal stories and make individualized responsibility attributions (Hook \& Pu, 2006; Kim et al., 2010). Additionally, heavier financial pressures on profit-oriented news organizations to attract a large number of news consumers may explain, from an organizational perspective, why narrative-style episodic framing was used in depression coverage to a greater extent in American than in Chinese newspapers.

These findings have practical implications for combating depression as a public health threat. In the case of Chinese newspapers, the coverage was overwhelmingly thematic (average framing rating was 4.89 on a scale of $1-5$, with higher scores indicating higher levels of thematic framing). While this framing approach may help facilitate overall issue understanding and alleviate victimblaming stigmas, the considerable lack of episodic framing in the form of personal storytelling, including the invisibility of individual sufferers in newspaper coverage, however, may inadvertently mitigate the effectiveness of depressionrelated communication, as research suggests that episodic framing can achieve exemplification effects in the processing of mediated information (Zillmann, 2002). Health journalists in China should consider balancing thematic and episodic framing to help the public understand the broader picture as well as the personal impact of depression as a public health issue. This may indeed be the direction that health journalism is already taking in China, as we also found an increase in the use of episodic framing in Chinese newspaper coverage of depression since 2000. This finding and its public opinion implications will be further elaborated on in our discussion of RQ2 results.

Compared with Chinese newspapers, US newspaper framing of depression was more episodic. While this framing approach may achieve some exemplification effects in reaching news audiences, it also has the potential to hinder overall issue understanding, responsibility attribution and stigma alleviation. Research has demonstrated that episodic framing encourages a "morselized" understanding of social issues by describing chronic problems as a series of discrete cases and idiosyncratic instances rather than deep-seated, interconnected and recurring conditions at societal and systemic levels (Iyengar, 1991, p. 136). This flawed understanding may divert public attention from societal responsibilities, undermine public support for government programs and other society-level solutions, and cause the public to blame mental disorder sufferers for their own predicaments. This framing tendency, however, was more pronounced in US local than in national newspapers, which we discuss in the following section. 


\section{More Thematic Framing in US National than Local Newspapers}

When comparing depression coverage in national and local newspapers, results suggest that organizational constraints may be another factor that needs to be considered when examining frame building in the news media. In this study, newspapers of national reach and stature in the US utilized more thematic framing in their depression coverage than local newspapers, possibly due to more resources in conducting research and collecting data as well as more access to national-level news sources. In the newsgathering process, journalists tend to rely on the usual suspects (e.g., experts and officials) rather than average citizens to help make sense of social realities and occurrences (Herman \& Chomsky, 2002). A complex health issue such as depression may be particularly prone to be dependent on explanations and interpretations from medical and professional experts. News stories built on materials provided by these sources are more likely to be thematic than episodic. National newspapers' heavier use of thematic framing may be related to their greater access to such sources, an organizational characteristic that sets them apart from local newspapers.

Another journalistic routine involves beat reporting and coverage of media events put forth by government institutions and/or public health advocacy groups, in which reporters again will likely resort to familiar and easy-to-access sources such as officials, experts, and media relations professionals. National newspapers in particular may allocate more coverage to large-scale public health advocacy and campaign efforts as well as government regulations and other policy-based programs, making thematic framing more likely to be used in national than in local newspapers. On the other hand, local newspapers' tendency to use local sources and cover local events, especially those of tragic nature with negative impact on local residents and communities, likely yielded more episodic framing in which personal tragedies and sufferings were highlighted or even dramatized. Considering that local news still tends to be perceived as a more credible source of health information by the general public (Holder \& Trenor, 1997), too much emphases on episodic framing is concerning and need to be addressed in future research as well as in health journalism practice and mental health communication in general.

\section{Gradual Increase in Episodic Framing in Chinese Newspapers}

As mentioned earlier, despite an overwhelming tendency of thematic framing in depression coverage, Chinese newspapers displayed a gradual increase in episodic framing from 2000 to 2012. Changes in cultural and organizational orientations may be some of the underlying contributing factors. As societies undergo modernization and industrialization, cultural orientations often shift from traditional collectivism to individualism, and Chinese culture may be no exception (Triandis, 1995). Since the late 1970s, the Chinese society experienced some of the most dramatic transformations in modern history, including those in political governance (more open and liberal albeit remaining the one-Party rule), economic structure (from centrally planned to market-oriented), and social conditions (e.g., 
urbanization and the emergence of a middle class) (Zhang, 2009). These systemic changes have had a profound impact on the Chinese culture, leading to the socalled "rediscovery of the individual" in which individual interests, plights and pursuits that used to be overlooked or even dismissed have now become increasingly visible in civic life ( $\mathrm{Li}, 2002)$. These cultural shifts seem to be reflected in the media. For example, China Central Television produced and aired shows with themes such as "telling stories of the ordinary people" and asking citizens "are you happy" on a personal level (Media.people.com.cn, 2012; News.cntv.cn, 2012). Under these circumstances, episodic framing which emphasizes personal storytelling may become more prevalent.

Meanwhile, organizational reconfigurations in the Chinese press during the reform era resulted in changes in editorial orientations, revenue-earning mechanisms, and journalism education, which may all contribute to the rise in episodic news framing. Increasingly cut-throat market competition and mounting commercial pressures on news organizations may lead to more trivialization and dramatization of serious news events and social issues, causing the decrease in thematic framing. Westernization of journalism education, on the other hand, may have produced a new generation of journalists who become exposed and receptive to Western news values and journalistic practices that emphasize human interest storytelling (Luther \& Zhou, 2005). On one hand, the emerging trend of episodic framing may enhance journalism's audience appeal and in turn news organizations' competitive edge in the increasingly congested Chinese media market. On the other hand, health journalists covering mental health issues in China may fall prey to the same fallacy made by their Western counterparts in deemphasizing the larger social dimensions and consequences of mental health problems, thereby doing a disservice to public understanding of the critical health issue of depression. As Chinese news organizations continue to find themselves in an ever-evolving cultural, social and media environment, future research should continue to monitor whether and to what extent the shift from thematic to episodic framing sustains over time and across media.

\section{Conclusions, Limitations and Future Research}

This study offered a comparative, longitudinal and empirical understanding of how depression, one of the most severe health threats to both the Chinese and the American population, has been thematically and episodically framed by a wide range of newspapers in the two countries from 2000 to 2012. Methodologically, we developed and validated a comprehensive and reliable measurement for the critical concept of thematic and episodic news framing in the context of depression coverage. Theoretically, we explored the under-investigated question of frame building, offering insights into how two macro-level factors, cultural values and organizational constraints, may have played a role in shaping the ways in which newspapers in China and the US framed the public health issue of depression. Our findings provided useful knowledge for health communication professionals and health journalists to better understand the status quo of health journalism practice as well as the trend of mental health information dissemination in both countries. 
We identified several future research directions to address limitations in the current study. First, while our findings suggested the influence of cultural values and organizational constraints as possible frame building factors, due to the limitations inherent in the content analysis methodology, more production- and process-oriented research (e.g., newsroom participant observations, in-depth interviews and surveys of news managers and reporters) needs to be conducted to directly assess the impact of various macro- and micro-level factors on news framing. Second, this study focused on news coverage within a relatively short timeframe (2000-2012) due to limitations in data availability. Future research extending study timeframe to the early 1980s when landmark events in both countries (e.g., the beginning of press reform in China and the start of a biomedical model of understanding for mental disorders in the US following the publication of DSM-III) may yield deeper understanding of how depression framing in the two countries has evolved as a function of changes in the larger socio-cultural environment. Additionally, updated research building on our study monitoring depression framing after 2012 can further advance theoretical understanding of the potential impact of various frame building factors that may have emerged in the fluctuating political, social, and media environment in recent years. Finally, the content, impact and effectiveness of various framing approaches in not only news coverage but also strategic communication such as public health campaigns and public service announcements associated with depression prevention, intervention, and treatment need to be examined via experimental design research.

\section{References}

Beaudoin, C. E. (2007). SARS news coverage and its determinants in China and the US. The International Communications Gazette, 69(6), 509-524.

CCTV-China Central Television (2015, Oct. 10). China helps depression patients by map technology. CCTV.Com. Retrieved from goo.gl/d9T8P8.

Census Regions and Divisions of the United States. (2012, Nov. 23). Census.gov. Retrieved from: goo.gl/i9QmY6.

Cho, S. (2006). Network news coverage of breast cancer, 1974 to 2003. Journalism \& Mass Communication Quarterly, 83(1), 116-130.

Conrad, P. (2001). Genetic optimism: Framing genes and mental illness in the news. Culture, Medicine and Psychiatry, 25(2), 225-247.

Depression. (2014, Apr. 26). Nimh.nih.gov. Retrieved from http://www.nimh.nih.

Dong, D., Chang, T., \& Chen, D. (2008). Reporting AIDS and the invisible victims in China: Official knowledge as news in the People's Daily, 1986-2002. Journal of Health Communication, 13, 357-374.

Dorfman, L., Woodruff, K., Chavez, V., \& Wallack, L. (1997). Youth and violence on local television news in California. American Journal of Public Health, 87(8), 1311-1316.

Dubriwny, T. N. (2010). Television news coverage of postpartum disorders and the politics of medicalization. Feminist Media Studies, 10(3), 285-303.

Dudo, A. D., Dahlstrom, M. F., \& Brossard, D. (2007). Reporting a potential pandemic. Science Communication, 28(4), 429-454 
Entman, R. M. (2004). Projections of power: Framing news, public opinion, and US foreign policy. Chicago, IL: University of Chicago Press.

Gans, H. (1979). Deciding what's news. New York, NY: Pantheon.

Gries P. H., \& Peng, K. (2002). Cultural clash? Apologies East and West. Journal of Contemporary China, 11(30), 173-178.

Gross, K. (2008). Framing persuasive appeals: Episodic and thematic framing, emotional response, and policy opinion. Political Psychology, 29(2), 169-192.

Guo, S., \& Guo, K. (2005). Research progress on depression. China Journal of Clinical Rehabilitation, 9(4), 131-133.

He, L., \& Wang, X. (2012). Depression communication in China. Medicine and Philosophy, 33(2A), 29-31 (in Chinese).

Herman, E. S., \& Chomsky, N. (2002). Manufacturing Consent: The Political Economy of the Mass Media. New York, NY: Pantheon.

Holder, H., \& Trenor, A. (1997). Media advocacy in community prevention: News as a means to advance policy change. Addiction, 92(Suppl. 2), S189-S199.

Hook, S. W., \& Pu, X (2006). Framing Sino-American relations under stress: A reexamination of news coverage of the 2001 spy plane crisis. Asian Affairs: American Review, 33(3), 167-183.

Iyengar, S. (1991). Is Anyone Responsible? How Television Frames Political Issue. Chicago, IL: University of Chicago Press.

Johansson, E. E., Bengs, C., Danielsson, U., Lehi, A., \& Hammarstrom, A. (2009). Gaps between patients, media and academic medicine in discourses on gender and depression: A metasynthesis. Qualitative Health Research, 19(5), 633-644.

Kim, S.-H., Carvalho, J. P., \& Davis, A. G. (2010). Talking about poverty: News framing of who is responsible for causing and fixing the problem. Journalism \& Mass Communication Quarterly, 87(3-4), 563-581.

Kim, S.-H., Carvalho, J. P., Davis, A. G., \& Mullins, A. M. (2011). The view of the border: News framing of the definition, causes, and solutions to illegal immigration. Mass Communication and Society, 14(3), 292-314.

Kim, S.-H., \& Willis, L. A. (2007). Talking about obesity: News framing of who is responsible for causing and fixing the problem. Journal of Health Communication, 12(4), 359-376.

Kleinman, A. (1986). Social origins of distress and disease: Depression, neurasthenia, and pain in modern China. New Haven, London: Yale University Press.

Kleinman, A. (1995). Writing at the margin: Disclosure between anthropology and medicine. Berkeley, CA: University of California Press.

Kunkel, D., Smith, S., Suding, P., \& Biley, E. (2006). Informative or not? Media coverage of child social policy issues. In A. Ben-Arieh \& R. M. George (Eds.), Indicators of Children's Well-being: Understanding Their Role, Usage, and Policy Influence (Social Indicators Research Series Vol. 2) (173-191). Dordrecht, the Netherlands: Springer.

Lawrence, R. G. (2004). Framing obesity: The evolution of news discourse on a public health issue. Harvard International Journal of Press/Politics, 9(2), 56-75.

Li, O., (2002). Pursuit for Modernity. Beijing, China: Sanlian Publishing.

Liu, B., Ley, B. L., \& Brewer, P. R. (2011). Breast cancer coverage in China and the United States: A comparative analysis of wire service news stories. Chinese Journal of Communication, 4(June), 182-197.

Luther, C. A., \& Zhou, X. (2005). Within the boundaries of politics: News framing of SARS in China and the United States. Journalism and Mass Communication Quarterly, 82(4), 857-872. 
Major, L. H. (2009). Breaking it to me harshly: The effects of intersecting news frames in lung cancer and obesity coverage. Journal of Health Communication: International Perspectives, 14(2), 174-188.

Mastin, T., Choi, J., Barboza, G. E., \& Post, L. (2007). Newspaper framing of elder abuse: It's not a family affair. Journalism and Mass Communication Quarterly, 84(4), 777-794.

Mao, P., Tang, Y., \& Cai, Z. (2003). Choice of care for depression patients and analysis of correlating factors. Chinese Journal of Mental Health, 17, 421-422.

Media.people.com.cn. (2012, Oct. 27). Retrieved from goo.gl/49rmno.

Metzl, J. M., \& Angel, J. (2004). Assessing the impact of SSRI antidepressants on popular notions of women's depressive illness. Social Science and Medicine, 58(3), 577-584.

Murray, C., \& Lopez, A. D. (1996). Global health statistics: A compendium of incidence, prevalence, and mortality estimates for over 200 conditions. Cambridge, MA: Harvard University Press.

News.cntv.cn. (2012, Oct. 27). Retrieved from goo.gl/CbfZ9m.

Peng, W., \& Tang, L. (2010). Health content in Chinese newspapers. Journal of Health Communication, 15, 695-711.

Price, V., Tewksbury, D., \& Powers, E. (1997). Switching trains of thought: The impact of news frames on readers' cognitive responses. Communication Research, 24, 481-506.

Ryder, A. G., Sun, J., Zhu, X., Yao, S., \& Chentsova-Dutton, Y. E. (2012). Depression in China: Integrating developmental psychopathology and cultural-clinical psychology. Journal of Clinical Child and Adolescent Psychology, 41(5), 682-694.

Scheufele, D. A. (1999). Framing as a theory of media effects. Journal of Communication, 49(1), 103-122.

Schnittker, J. (2008). An uncertain revolution: Why the rise of a genetic model of mental illness has not increased tolerance. Social Science \& Medicine, 67(9), 1370-1381.

Shah, D. V., Kwak, N., Schmierbach, M., \& Zubric, J. (2004). The interplay of news frames on cognitive complexity. Human Communication Research, 30, 102-120.

Shoemaker, P. J., \& Reese, S. D. (1996). Mediating the message. White Plains, NY: Longman.

Stats and Facts about Depression in America. (2015, Aug. 19). Everydayhealth.com. Retrieved from goo.gl/ZZqIjs.

Stout, P. A., Villegas, J., \& Jennings, N. A. (2004). Images of mental illness in the media: identifying gaps in the research. Schizophrenia Bulletin, 30(3), 543-561.

Tan, Y. (2004). Current situation of public mental health in China and public policies. China Public Health Administration, 6, 364-366.

Tian, X., \& Cheng, W. (2014). From melancholia to depression: In the socio-cultural views of disease. Medicine and Philosophy, 35(2A), 79-81.

Triandis, H.C. (1995). Individualism and collectivism: New directions in social psychology. Boulder, CO: Westview Press.

Wang, P.S., Lane, M., Olfson, M., Pincus, H. A., Wells, K. B, \& Kessler, R. C. (2005). Twelve month use of mental health services in the United States. Archives of General Psychiatry, 62, 629-640.

Walker, I., \& Read, J. (2002). The differential effectiveness of psychosocial and biogenetic causal explanation in reducing negative attitudes toward "mental illness." Psychiatry, 65(4), 313-325. 
Wang, X. (2007). For the good of public health or for political propaganda? People's Daily's coverage of the Severe Acute Respiratory Syndrome epidemic. China Media Research, 3(3), 25-32.

Zhang, Y. (2009). Individualism or collectivism: Cultural orientations in Chinese TV advertising and analysis of some moderating factors. Journalism and Mass Communication Quarterly, 86(3), 630-653.

Zhao, Z. (2009). Investigation of the current status and future development of depression in China. Clinical Psychiatry, 19, 63-64.

Zillmann, D. (2002). Exemplification theory of media influence. In J. Bryant \& D. Zillmann (Eds.), Media effects: Advances in theory and research (19-41). Mahwah, NJ: Lawrence Erlbaum Associates. 